\title{
A search for the ideal plasmonic nanoshell: the effects of surface scattering and alternatives to gold and silver
}

\author{
Martin G. Blaber, Matthew D. Arnold and Michael J. Ford* \\ Department of Physics and Advanced Materials and Institute for Nanoscale Technology, University of \\ Technology, Sydney, 15 Broadway, Sydney 2007, NSW
}

mike.ford@uts.edu.au

\section{RECEIVED DATE}

The optical absorption efficiency of nanospheres and nanoshells of the elements $\mathrm{Na}, \mathrm{K}, \mathrm{Al} \mathrm{Ag}$ and $\mathrm{Au}$ are compared, and the effects of surface scattering, as introduced by the billiard model [Moroz, 2008] are discussed. We find that the introduction of surface scattering has comparatively little effect on the optimized absorption efficiency of nanospheres, with the maximum absorption efficiency of $\mathrm{K}$ nanospheres falling from 14.7 to 13.3. Conversely, the reduction in absorption efficiency in nanoshells is substantial. This effect is compounded in metals with higher plasma frequency. We show that the high comparative plasma frequencies in silver and gold results in a greatly reduced optimized absorption efficiency when compared to nanoshells in the absence of surface scattering. Whereas sodium and potassium, with low plasma frequencies, are not effected as much.

Plasmonics has applications in a variety of fields including super resolution planar lens lithography ${ }^{1-3}$, medical therapeutics and diagnostics technologies ${ }^{4-7}$, subwavelength color imaging systems ${ }^{8}$, high 
throughput communications and processing systems ${ }^{9}$, portable extreme UV lasers ${ }^{10}$ and solar glazing for energy efficient windows ${ }^{11}$. Most of these technologies rely on the strength and position of the surface plasmon on nanorods, nanoshells or nanotriangles and the fact that the nanoparticle will absorb incident light well at the resonance wavelength. The optical properties of nanorods and nanoshells have previously been compared ${ }^{12-14}$. In both cases the plasmon resonance can be tuned across a wide wavelength range by changing the aspect ratio. Gold nanorods give a larger absorption per unit volume, however, nanoshells have the distinct advantage that their optical properties are both orientation and polarization independent. Also, although the most efficient way of making nanorods ${ }^{15}$ and nanoshells ${ }^{16}$ is via wet chemistry, nanoshells or nanocaps can be made by evaporation or sputtering of the metal onto polystyrene spheres ${ }^{17,18}$. Production of nanorods in this fashion requires a more complex electrochemical templating technique ${ }^{19}$. A similar alumina templating technique applicable to reactive metals produces short, defective tubes rather than rods ${ }^{20}$. Considerable progress has been made in the application of gold nanoshells in thermal therapeutic treatment of cancer ${ }^{21}$.

Previously, we have investigated the merit of different metal nanospheres for use in plasmonics ${ }^{22}$. An analysis of 26 different, alkali, noble and transition metals showed that although gold and silver are the most commonly used metals for plasmonics, they are by far sub-optimal. The question addressed here is how this extends to metal nanoshells, particularly if the influence of surface scattering is taken into account. A rule of thumb is often used in connection with surface scattering from solid gold spheres where the effect becomes appreciable for spheres below about 5nm radius, experimental data support this assertion $^{23}$. For shells, however, the optimum geometry may involve a very thin shell where surface scattering can become important.

Many metals have limited applicability as plasmonic materials due to their chemical reactivity. The alkali metals are prime examples. The absorption efficiencies of alkali metal nanospheres are substantially higher than those of the noble metals, but they have had limited practical application, at 
least to date, due to their extreme reactivity. However, applications such as optical nanocircuits, solar glazings and UV lasers where direct contact with water or other oxidizing agents does not occur may provide situations where a passivating layer can be used.

In this paper we investigate, using Mie theory, the optical absorption of nanoshells composed of various metallic elements in order to identify the optimum geometry and material taking into account surface scattering using the simple billiard ball scattering model of Moroz ${ }^{24}$.

We use the program BHCOAT $^{25}$, a numerical solution to Mie theory for multiply coated spheres to calculate the absorption efficiency, $Q_{a b s}$, of nanospheres and nanoshells. Bulk dielectric constants were taken from Weaver and Frederikse ${ }^{26}$. The spheres and shells were embedded in vacuum and shell cores were also vacuum. Shell geometries were sampled with a Gaussian distribution designed to include a large number of steps in the vicinity of the solid sphere limit (aspect ratio 0) and the infinitely thin shell limit (aspect ratio 1), with comparatively few points in between. We have included the full multipole expansion of Mie theory to ensure that symmetric and anti-symmetric multipole modes contribute to the spectra.

For structures with small aspect ratios, that is shells with a thickness of a few nanometers or spheres of similar diameter, the mean free path of the electrons is comparable to this critical size. Surface scattering can then reduce the absorption efficiency and broaden the plasmon resonance. We have included this effect in our model using the recently derived billiard scattering model by Moroz $^{24}$ to modify the bulk dielectric. This model provides a method for calculating the damping term associated with surface scattering, but requires invoking an analytical model for the dielectric function in order to modify the experimental bulk dielectric. We follow the commonly used method of permittivity augmentation $^{27,28}$ where a Drude model is used to incorporate this additional damping but is applied in such a way that interband transitions are not neglected. 
The intraband contribution to the permittivity can be described by the Drude model

$$
\varepsilon_{D}\left(\gamma_{b u l k}\right)=1-\frac{\omega_{p}^{2}}{\omega\left(\omega+i \gamma_{b u l k}\right)}
$$

where $\omega_{\mathrm{p}}$ the bulk plasma frequency, and $\gamma_{\text {bulk }}$ the bulk damping (inverse of the relaxation time $\tau$ ).

Using the experimentally determined values for $\omega_{\mathrm{p}}$ and $\gamma_{\text {bulk }}$ given in table 1 we can calculate this intraband component, and by adding the damping, $\gamma_{\text {surf }}$, from the billiard model correct this for surface scattering. The surface scattering modified experimental dielectric, $\varepsilon_{C}$, which includes both the intraand interband components is then

$$
\varepsilon_{\mathrm{C}}=\varepsilon_{\exp }+\Delta \varepsilon=\varepsilon_{\text {exp }}+\varepsilon_{\mathrm{D}}\left(\gamma_{\text {bulk }}+\gamma_{\text {surf }}\right)-\varepsilon_{\mathrm{D}}\left(\gamma_{\text {bulk }}\right)
$$

Table. 1. Optical data used for the addition of billiard scattering to the bulk experimental permittivity.

\begin{tabular}{llll}
\hline Element & $\omega_{\mathrm{p}}(\mathrm{eV})$ & $\gamma_{\text {intra }}(\mathrm{eV})$ & $V_{F}\left(10^{6} \mathrm{~m} / \mathrm{s}\right)^{29}$ \\
\hline $\mathrm{Ag}$ & $9.6^{30}$ & $0.0228^{31}$ & 1.39 \\
$\mathrm{Au}$ & $8.55^{30}$ & $0.0184^{31}$ & 1.40 \\
$\mathrm{Al}$ & $15.3^{32}$ & $0.5984^{33}$ & 2.03 \\
$\mathrm{Na}$ & $5.71^{32}$ & $0.0276^{34}$ & 1.07 \\
$\mathrm{~K}$ & $3.72^{32}$ & $0.0184^{34}$ & 0.86 \\
\hline
\end{tabular}

Damping due to surface scattering is calculated from

$$
\gamma_{\mathrm{S}}=\frac{V_{\mathrm{F}}}{L_{\mathrm{B}}}
$$

where $V_{\mathrm{f}}$ is the Fermi velocity, given in table 1 , and $L_{\mathrm{B}}$ is the mean free path of the electrons in the shell, calculated from ${ }^{24}$

$$
L_{\mathrm{B}}=\frac{4\left(r_{\mathrm{o}}^{3}-r_{\mathrm{i}}^{3}\right)}{3\left(r_{\mathrm{o}}^{2}+r_{\mathrm{i}}^{2}\right)},
$$


where $r_{o}$ is the outer radius and $r_{i}$ is the inner radius. Moroz derives the mean free path of an electron in the billiard scattering model where reflections from the internal surfaces of the shell are specular and reports that not only does it match the experimental results for nanoshells but reduces to the well-known result for a sphere of $4 r / 3$.

The correction method described above is limited in some circumstances. If the bulk damping is large, or for long wavelengths, the damping maybe greater than the frequency in the Drude expression (1). Additional damping then causes the surface scattering corrected imaginary permittivity to be reduced resulting in unphysically large absorption. This happens in aluminum at long wavelengths and so we restrict ourselves to shells thicker than $0.8 \mathrm{~nm}$.

We have previously reported the optimum geometries for solid spheres of various metallic elements excluding surface scattering ${ }^{22}$. Most notably, gold has a maximum absorption efficiency of 3.3 for a sphere radius of $49 \mathrm{~nm}$, whereas potassium reaches a maximum of 14.7 for a $21.3 \mathrm{~nm}$ radius sphere. Gold is far from the optimal material for spheres, at least as far as achieving maximum absorption is concerned. The inclusion of surface scattering has little effect because of the large mean free path $4 / 3 r_{0}$. Even in aluminum, where the optimum size is approximately $6 \mathrm{~nm}$ radius, our calculation of surface scattering has little influence. The intraband damping in aluminum is so large that the additional damping due to surface scattering only causes the optimized absorption efficiency to be reduced from 13.1 to 10.9 .

To optimize the geometry of the nanoshells it is necessary to calculate the absorption efficiency as a function of aspect ratio and overall shell diameter. Here we calculate the optimum aspect ratio for outer radii in the range 1.0 to $70.0 \mathrm{~nm}$, the results are given in table 2 both with and without the inclusion of surface scattering, Figure 1 shows the associated absorption spectra. 
Table 2. Shell geometries for optimized absorption with and without surface scattering

\begin{tabular}{|c|c|c|c|c|c|c|}
\hline \multirow[b]{2}{*}{ Element } & \multicolumn{3}{|c|}{ No Surface Scattering } & \multicolumn{3}{|c|}{ With Surface Scattering } \\
\hline & $Q_{\text {abs }}$ & $\begin{array}{l}\text { Outer } \\
\text { Radius } \\
(\mathrm{nm})\end{array}$ & $\begin{array}{l}\text { Inner } \\
\text { Radius } \\
\text { (nm) }\end{array}$ & $Q_{\text {abs }}$ & $\begin{array}{l}\text { Outer } \\
\text { Radius } \\
(\mathrm{nm})\end{array}$ & $\begin{array}{l}\text { Inner } \\
\text { Radius } \\
(\mathrm{nm})\end{array}$ \\
\hline $\mathrm{Ag}$ & 12.2 & 57.4 & 56.5 & 5.7 & 32.8 & 27.2 \\
\hline $\mathrm{Au}$ & 19.9 & 29.2 & 27.7 & 7.3 & 42.2 & 37.5 \\
\hline $\mathrm{Al}$ & 13.1 & 5.8 & 0.0 & 10.9 & 6.6 & 0.0 \\
\hline $\mathrm{Na}$ & 20.6 & 27.8 & 24.8 & 11.4 & 29.8 & 23.0 \\
\hline $\mathrm{K}$ & 22.8 & 35.0 & 30.7 & 13.7 & 36.0 & 27.2 \\
\hline
\end{tabular}
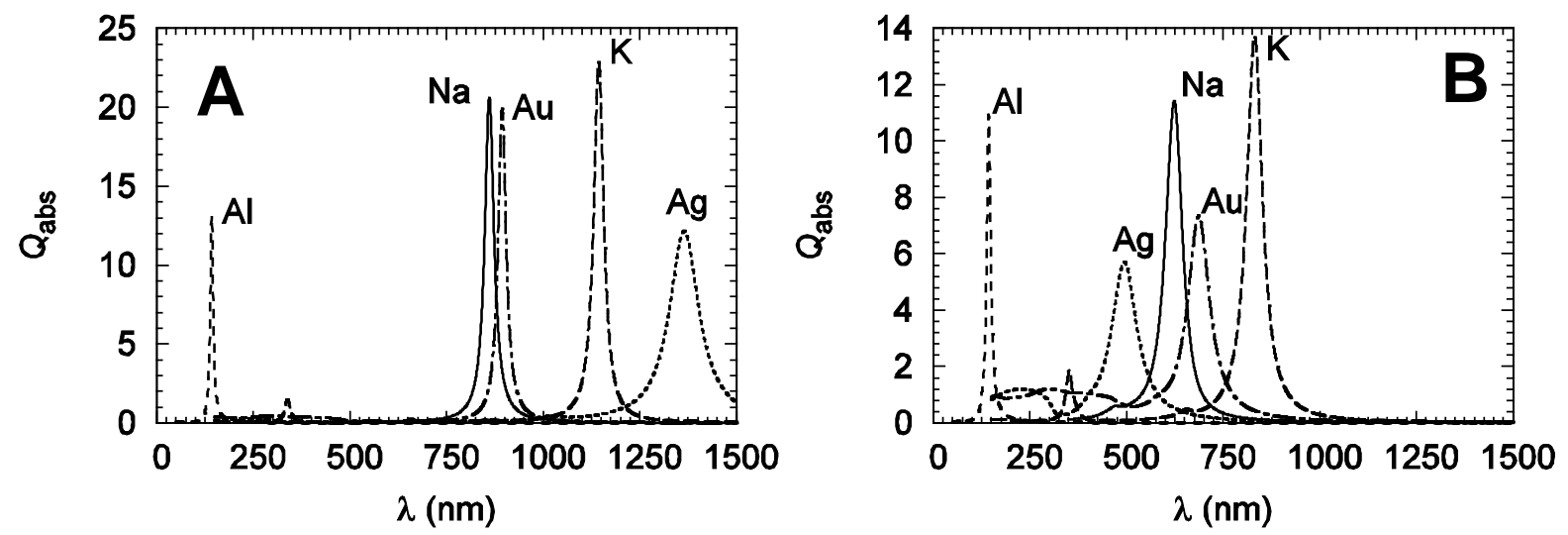

Figure 1. Optimized absorption spectra for nanoshells with radii and aspect ratios given by Table 2 (a) without surface scattering and (b) with surface scattering.

Without including surface scattering, $\mathrm{K}$ has the best $Q_{a b s}$ with a value of 22.8 at a wavelength of 1144 nm, followed closely by sodium and gold. and then by silver and aluminum. For the sodium and potassium shells, the increase in absorption efficiency, over that of the optimized sphere, is about $160 \%$. For silver, it is just over $200 \%$ and for gold the increase is a remarkable $600 \%$. This increase in the absorption efficiency for gold is due to the position of the band-edge at $2.25 \mathrm{eV}$ which means the interband transitions are close to the resonance position for the solid sphere $\left(\varepsilon^{\prime}=-2\right)$ and hence decrease the absorption efficiency. For a shell, the resonance condition shifts to longer wavelengths, away from 
the interband transitions, as the aspect ratio decreases. Coupled with a low intraband damping this means the absorption efficiency can increase dramatically with increasing wavelength. For silver, the band edge is at higher frequency $(3.9 \mathrm{eV})$ and does not contribute to damping for the sphere resonance, in addition intraband damping at longer wavelengths is larger and the absorption gain from sphere to nanoshell is not large. This result can be visualized by plotting the quality factor determining the strength of the resonance, $-\varepsilon^{\prime} / \varepsilon^{\prime \prime}$, as a function of wavelength. This is shown in Figure 2 and is the figure of merit for nanoshells in the absence of surface scattering for the various metallic elements considered here. $\mathrm{Na}, \mathrm{K}$ and $\mathrm{Au}$ all perform reasonably well from about $800 \mathrm{~nm}$ into the infrared, this is assuming that a suitable shell can be made to access these long wavelength portions of the permittivity, in reality the shell may be so thin that surface scattering effects diminish the resonance.

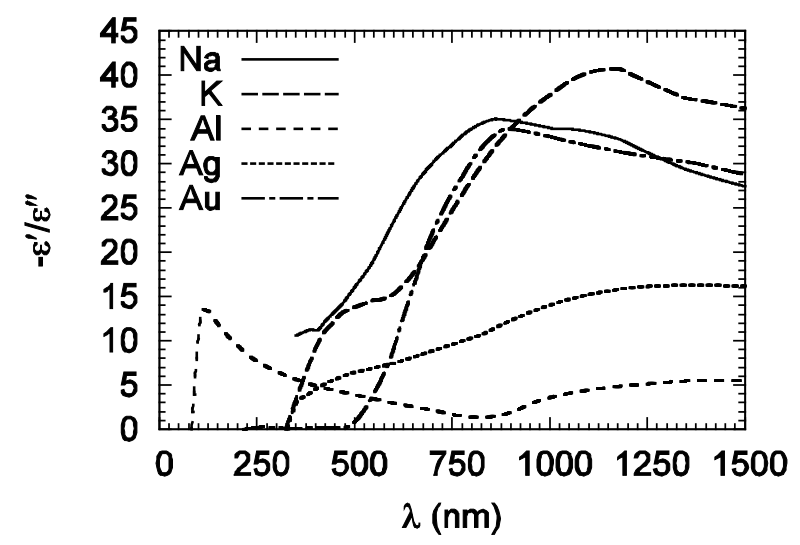

Figure 2. The quality factor $-\varepsilon^{\prime} / \varepsilon^{\prime \prime}$ from experimental optical data. The best metal is potassium, followed by sodium, gold, silver and aluminum.

The optimum geometry for an Aluminum nanoshell is just the sphere (Table 2 and Figure 1), due to the relationship between the real and imaginary parts of the permittivity. In the limit of no interband transitions, and for frequencies below the plasma frequency, we can approximate the $-\varepsilon^{\prime} / \varepsilon^{\prime \prime}$ relationship with $\omega / \gamma$, indicating that for a Drude-like metal, the best resonance will occur at the highest possible 
frequency. For aluminum, the best $-\varepsilon^{\prime} / \varepsilon^{\prime \prime}$ ratio occurs at $112 \mathrm{~nm}$, which is below the threshold wavelength for the excitation of a resonance (as $\varepsilon^{\prime}=-0.93$ ).
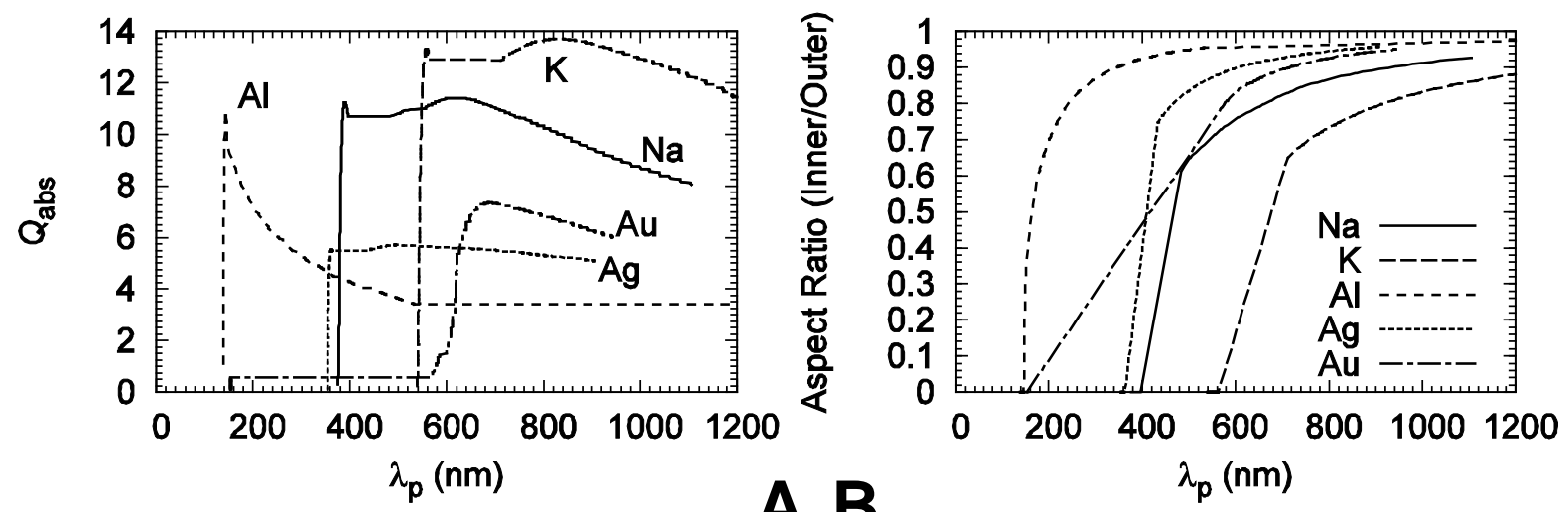

A B

$C D_{14}$
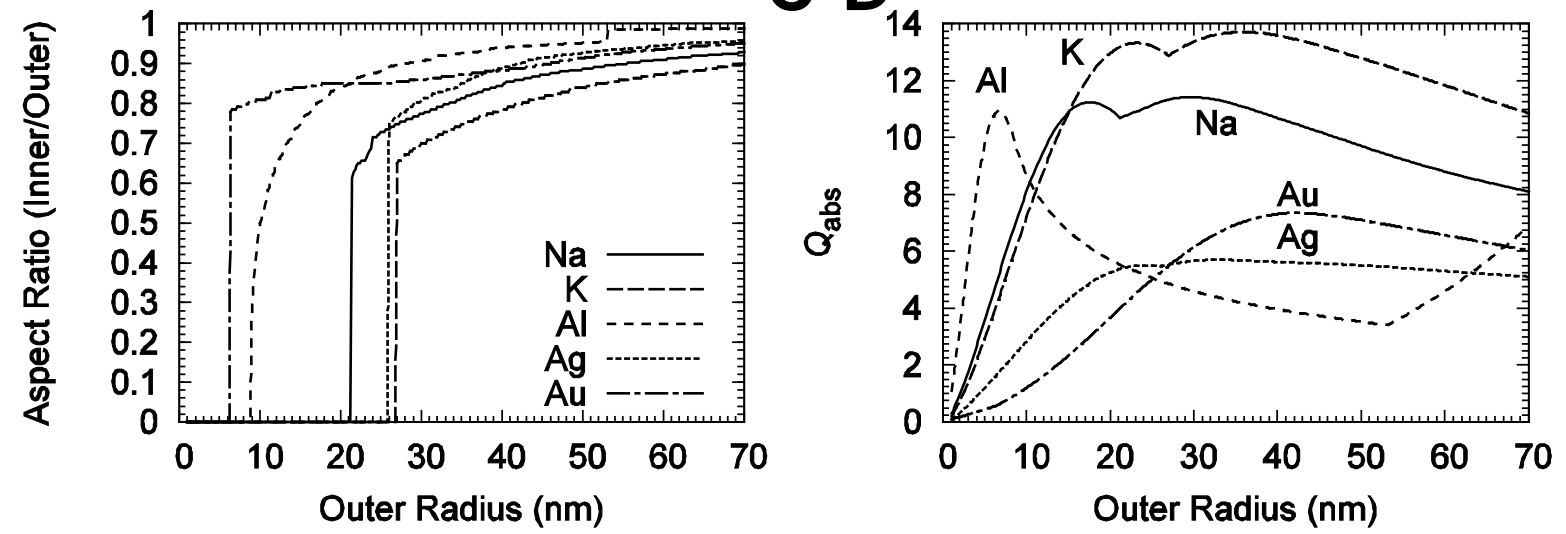

Figure 3. Optimized absorption efficiency for nanoshells including surface scattering. A) The optimum absorption efficiency as a function of wavelength. B) The aspect ratio that gives the optimum resonance in A. C) The aspect ratio at which the resonance occurs as a function of Outer radius. D) Absorption efficiency of the resonance with optimized aspect ratio and wavelength.

From the above discussion it is clear that surface scattering corrections need to be applied in order to understand the response of nanoshells at longer wavelengths where the shell can be quite thin. Figure 3 shows the results of optimization calculations with the bulk dielectric corrected for surface scattering. In each case an outer radius is chosen then the optimum aspect ratio is determined for that outer radius. 
Figure 3 shows this data, aspect ratio is the ratio of inner to outer shell radius, zero represents a solid shell and one an infinitesimally thin shell.

The sharp edges present in the data of Figure 3(a) reflects the point at which a solid sphere gives the optimum absorption efficiency, and correspond to the point at which the aspect ratio becomes zero in Figure 3(b). The plot of absorption versus wavelength is very steep at this point because the plasmon resonance of a sphere does not shift very much with sphere size. This effect can also be seen in Figure 3(d) particularly for $\mathrm{Na}$ and $\mathrm{K}$ where there is a noticeable cusp in the data at around 20 and $30 \mathrm{~nm}$ outer radius respectively. Au is different because for quite small outer radii, less than about $10 \mathrm{~nm}$ single electron interband transitions dominate the absorption. This is why the absorption in Au extends down to wavelengths shorter than $200 \mathrm{~nm}$ when the plasmon resonance of the sphere is only about $530 \mathrm{~nm}$.

The inclusion of surface scattering has reduced the optimum absorption efficiency of sodium and potassium nanoshells by $45 \%$ and $40 \%$ respectively, a large improvement over the reduction in silver and gold which stand at 53\% and 63\% respectively. From figure 3(a) Na provides the best absorption over the shorter wavelength visible region and K over the red to near-infrared region. The aspects ratios of the corresponding shells are also reasonable within these regimes for these two elements (figure 3(b)) with $\mathrm{Na}$ being less than about 0.7 and $\mathrm{K}$ less than 0.9. By contrast the aspect ratios in gold or silver required to access these regions are greater than 0.9 . This is the reason why these two elements do not perform well, they are limited by surface scattering.

The absorption efficiency of Aluminum nanoshells has shifted extensively into the infrared in the large radius limit upon the inclusion of surface scattering. This appears counterintuitive as not only have we restricted the shell thickness to $0.8 \mathrm{~nm}$, but also because the other metal nanoshell resonances are blue shifted when surface scattering is included. However, the red-shift is a general function of the low energy interband transition that disrupts the $\varepsilon^{\prime \prime}$ spectrum of aluminum. The overall effect of the 
transition runs from $400 \mathrm{~nm}$ to $1100 \mathrm{~nm}$, which causes the resonance to make a jump across a range of wavelengths. This jump requires a dramatic reduction in the shell thickness, furthermore, the resonance enters the long wavelength regime and the previously discussed anomalous absorption effect starts to appear, driving an increase in the absorption efficiency for shells with $r_{0}>53.0 \mathrm{~nm}$ (see Figure $3 \mathrm{C}, \mathrm{D}$ )

We have used Mie Theory and the Billiard scattering model to calculate the optimized absorption efficiencies of nanospheres and vacuum core - metal shell nanoparticles made of aluminum, sodium, potassium, silver and gold.

The effect of the introduction of surface scattering (using the Billiard scattering model) on optimized solid nanospheres has shown to be small, with aluminum spheres suffering a $17 \%$ reduction in absorption efficiency, and the other metals suffering less than $10 \%$ reduction in $Q_{a b s}$, with minor red shifting of the position of the resonance to allow for larger spheres. Potassium metal spheres had the highest absorption efficiency, over 400\% larger than that of the optimized gold nanosphere.

The effect of surface scattering is more profound on nanoshells, with all the optimized resonances blue-shifting to compensate for a general increase in shell thickness. This effect resulted in a bias towards low plasma frequency metals as $-\partial \varepsilon^{\prime} / \partial \lambda$ is close to zero, resulting in a tunability across the spectrum that enables the resonance to be placed in the (usually high wavelength) $-\varepsilon^{\prime} / \varepsilon^{\prime \prime}$ maximum, without a huge decrease in the shell thickness. We showed that for a Drude metal, the optimum geometry is a sphere as $-\varepsilon^{\prime} / \varepsilon^{\prime \prime} \approx \gamma / \omega$, however, interband transitions usually disrupt this. The introduction of surface scattering for aluminum nanoshells was troublesome, as additional damping from surface scattering resulted in a negative correction to the imaginary part of the permittivity, resulting in anomalously high absorption efficiency. Silver and gold nanospheres, suffered a reduction in absorption efficiency due to high plasma frequencies that caused the resonances to blue-shift away from the $-\varepsilon^{\prime} / \varepsilon^{\prime \prime}$ maximum. 
Sodium and potassium nanoshells offer a considerable maximum absorption efficiency advantage over both silver and gold nanoshells. Offering up to an $86 \%$ increase over the $Q_{a b s}$ of gold nanoshells and a $240 \%$ increase over silver. This dramatic increase may be worth the additional inconveniencies of handling these reactive metals.

\section{ACKNOWLEDGMENT}

This work was supported by the Australian Research Council, and the University of Technology, Sydney. Computing resources were provided by the Australian Centre for Advanced Computing and Communication (ac3) in New South Wales and the National Facility at the Australian Partnership for Advanced Computing (APAC).

References

1. Melville, D. O. S.; Blaikie, R. J. Opt. Express 2005, 13, (6), 2127-2134.

2. Fang, N.; Lee, H.; Sun, C.; Zhang, X. Science 2005, 308, (5721), 534-537.

3. Blaber, M. G.; Arnold, M. D.; Harris, N.; Ford, M. J.; Cortie, M. B. Physica B 2007, 394, (2), 184-187.

4. Hirsch, L. R.; Stafford, R. J.; Bankson, J. A.; Sershen, S. R.; Rivera, B.; Price, R. E.; Hazle, J. D.; Halas, N. J.; West, J. L. Proc. Nat. Acad. Sci 2003, 100, 13549.

5. Pissuwan, D.; Valenzuela, S. M.; Cortie, M. B. Trends Biotechnol. 2006, 24, (2), 62-67.

6. Pissuwan, D.; Valenzuela, S. M.; Killingsworth, M. C.; Xu, X. D.; Cortie, M. B. J. Nanopart. Res. 2007, 9, (6), 1109-1124.

7. Pissuwan, D.; Valenzuela, S. M.; Miller, C. M.; Cortie, M. B. Nano Lett. 2007, 7, (12), 38083812. 
8. Kawata, S.; Ono, A.; Verma, P. Nat. Photonics 2008, advanced online publication.

9. $\quad$ Engheta, N. Science 2007, 317, (5845), 1698-1702.

10. Kim, S.; Jin, J.; Kim, Y.-J.; Park, I.-Y.; Kim, Y.; Kim, S.-W. Nature 2008, 453, (7196), 757-760.

11. Cortie, M.; Xu, X.; Chowdhury, H.; Zareie, H.; Smith, G. Proc. SPIE 2005, 5649, 565.

12. Harris, N.; Ford, M. J.; Cortie, M. B. J. Phys. Chem. B 2006, 110, (22), 10701-10707.

13. Harris, N.; Ford, M. J.; Mulvaney, P.; Cortie, M. B. Gold Bulletin 2008.

14. Jain, P. K.; Lee, K. S.; El-Sayed, I. H.; El-Sayed, M. A. J. Phys. Chem. B 2006, 110, (14), 72387248.

15. Nikoobakht, B.; El-Sayed, M. A. Chem. Mater. 2003, 15, 1957-1962.

16. Sun, Y. G.; Mayers, B. T.; Xia, Y. N. Nano Letters 2002, 2, (5), 481-485.

17. Liu, J. Q.; Cankurtaran, B.; Wieczorek, L.; Ford, M. J.; Cortie, M. Adv. Funct. Mater. 2006, 16, (11), 1457-1461.

18. Cortie, M. B.; Dowd, A.; Harris, N.; Ford, M. J. J. Phys. Rev. B: Condens. Matter 2007, 75, (11), 113405.

19. van der Zande, B. M. I.; Böhmer, M. R.; Fokkink, L. G. J.; Schönenberger, C. Langmuir 2000, 16, 451-458.

20. Gentle, A.; Maaroof, A.; Smith, G.; Cortie, M. Proc. SPIE 2006, 6038, 603816.

21. Loo, C.; Lowery, A.; Halas, N.; West, J.; Drezek, R. Nano Lett. 2005, 5, (4), 709-711.

22. Blaber, M.; Harris, N.; Ford, M. J.; Cortie, M. B. In Nanoscience and Nanotechnology, 2006. ICONN '06. International Conference on, Proc. IEEE, 2006; 2006; p 561. 
23. Berciaud, S.; Cognet, L.; Tamarat, P.; Lounis, B. Nano Lett. 2005, 5, (3), 515-518.

24. Moroz, A. J. Phys. Chem. C 2008, 112, (29), 10641-10652.

25. Bohren, C. F.; Huffman, D. R., Absorption and scattering of light by small particles. Wiley: Weinheim, 2004.

26. Weaver, J. H.; Frederikse, H. P. R., Optical properties of selected elements 82 ed. CRC Press, Boca Raton, FL: 2001.

27. Nehl, C. L.; Grady, N. K.; Goodrich, G. P.; Tam, F.; Halas, N. J.; Hafner, J. H. Nano Lett. 2004, 4, (12), 2355-2359.

28. Pena, O.; Pal, U.; Rodriguez-Fernandez, L.; Crespo-Sosa, A. J. Opt. Soc. Am. B: Opt. Phys. 2008, 25, (8), 1371-1379.

29. Ashcroft; Mermin, Solid State Physics. College Edition ed.; Saunders College Publishing: 1976.

30. Noguez, C.; Román-Velázquez, C. E. Phys. Rev. B Condens. Matt. 2004, 70, (19), 195412.

31. Link, S.; El-Sayed, M. A. Int. Rev. Phys. Chem. 2000, 19, (3), 409-453.

32. Kittel, C., Introduction to Solid State Physics. Seventh ed.; John Wiley \& Sons: New York, 1996.

33. LaVilla, R.; Mendlowitz, H. Phys. Rev. Lett. 1962, 9, (4), 149.

34. Smith, N. V.; Spicer, W. E. Phys. Rev 1969, 188, (2), 593. 